\title{
Making heads or tails of mitochondrial membranes in longevity and aging: a role for comparative studies
}

\author{
Teresa G Valencak ${ }^{1,2^{*}+}$ and Vian Azzu 3 $^{* \dagger}$
}

\begin{abstract}
Mitochondria play vital roles in metabolic energy transduction, intermediate molecule metabolism, metal ion homeostasis, programmed cell death and regulation of the production of reactive oxygen species. As a result of their broad range of functions, mitochondria have been strongly implicated in aging and longevity. Numerous studies show that aging and decreased lifespan are also associated with high reactive oxygen species production by mitochondria, increased mitochondrial DNA and protein damage, and with changes in the fatty acid composition of mitochondrial membranes. It is possible that the extent of fatty acid unsaturation of the mitochondrial membrane determines susceptibility to lipid oxidative damage and downstream protein and genome toxicity, thereby acting as a determinant of aging and lifespan. Reviewing the vast number of comparative studies on mitochondrial membrane composition, metabolism and lifespan reveals some evidence that lipid unsaturation ratios may correlate with lifespan. However, we caution against simply relating these two traits. They may be correlative but have no functional relation. We discuss an important methodology for body mass and phylogenetic correction in comparative studies.
\end{abstract}

Keywords: Mitochondria, comparative biology, endotherm, ectotherm, fatty acid membrane composition, longevity, maximum lifespan

\section{Review}

\section{Introduction}

\section{A brief history of longevity hypotheses}

Over a century ago, Max Rubner observed for six animal species that larger animals had a slower metabolic rate per unit mass and a longer lifespan compared with smaller animals. Rubner [1] Later work by Kleiber [2] and others in the 1930s supported this finding for a larger range of species. This led to several hypotheses suggesting that aging and longevity are processes that are regulated by metabolic rate.

Raymond Pearl suggested that animal tissues had a finite number of chemical reactions available, which on exhaustion led to mortality [3]. Therefore, organisms

\footnotetext{
* Correspondence: teresa.valencak@vetmeduni.ac.at; va@mrc-mbu.cam.ac.uk ${ }^{\dagger}$ Equal contributors

${ }^{1}$ Research Institute of Wildlife Ecology, Veterinary University Vienna, Savoyenstrasse 1, 1160 Vienna, Austria

${ }^{3}$ Christ's College \& Department of Medicine, University of Cambridge, Cambridge CB2 3BU, UK
}

Full list of author information is available at the end of the article with a higher metabolism per unit mass would age and die sooner. This became known as the 'rate of living hypothesis'.

As scientists were just beginning to understand free radical biology in the 1950s [4], Denham Harman suggested a mechanism linking metabolic rate to aging and lifespan [5]. He proposed that reactive oxygen species, being the products of metabolism, would cause cumulative damage and result in aging followed by death. This 'free radical hypothesis of aging' actually echoed suggestions made earlier in the century by Elie Metchnikoff that 'senility' may be a consequence of 'waste' products of metabolism [6].

Studies showing that metabolic rate-matched [7] or size-matched animals had different lifespans $[7,8]$ undermined the rate of living hypothesis and suggested that metabolic rate is not the exclusive determinant of lifespan. However, these early observations contributed to the question of why metabolic rate varies substantially across species, especially between size-matched endotherms 
(higher metabolic rate) and ectotherms (lower metabolic rate) [7]. Brand and colleagues examined metabolic rate differences in hepatocytes isolated from a mammal (a rat) and a reptile (a lizard) [9], and found that the respiration rate was fivefold higher in rat hepatocytes, possibly due to an increased amount of n-3 polyunsaturated fatty acid (PUFA) in the mitochondrial membranes [9]. However, they noted no difference in the percentage of respiration rate dedicated to processes such ATP production, proton leak across the mitochondrial inner membrane and maintenance of $\mathrm{Na} / \mathrm{K}$ antiporter activity at the plasma membrane [9]. The variation in amplitude but not distribution of metabolic rate across species and its correlation with mitochondrial phospholipid composition [10], led Hulbert and Else to propose that membrane composition acts as a 'pacemaker for metabolism' [11]. Specifically, they postulated that membrane polyunsaturation, higher in the tissues of mammals in comparison with reptiles, would increase the molecular activity of membrane proteins thereby increasing cellular metabolic activity. Although this hypothesis held true between some species, it did not when birds were introduced into the equation, as birds have an increased metabolic rate compared to mammals, but lower membrane polyunsaturation [12].

Because of the broad but not perfect correlations of membrane fatty acid levels with metabolism, and metabolism with lifespan, a natural line of investigation developed looking at membrane composition with respect to lifespan, thus developing into the 'homeoviscous-longevity adaptation' [13] and later, the 'membrane pacemaker hypothesis of aging' [14]. These hypotheses linked membrane fatty acid unsaturation to susceptibility to oxidative damage, the propagation of which is associated with aging and mortality. In light of an increasing number of studies that support and conflict with these hypotheses, our review seeks to explore the evidence for the link between mitochondrial phospholipid and fatty acid composition, metabolism and lifespan. We discuss the roles for allometric (body size) and phylogenetic (species relatedness) corrections when making comparisons between different species $[15,16]$.

\section{Membrane landscapes in mitochondria}

Mitochondria are intracellular organelles whose primary function is metabolic energy transduction and ATP synthesis. They also play vital roles in intermediate molecule metabolism, metal ion (calcium and iron) homeostasis, programmed cell death and regulation of the production of reactive oxygen species (ROS) [17]. As a result of their broad range of functions, mitochondria have been strongly implicated in aging and longevity (reviewed in [18]). In fact, numerous studies have shown that longevity or lifespan may be affected by mitochondrial ROS production [5], mitochondrial DNA damage [19] and mitochondrial membrane fatty acid composition [11], the latter will be the focus of this article.

\section{Background to phospholipids and fatty acids}

Membrane lipids can broadly be classified as glycerophospholipids, sphingolipids or sterols. These lipid moieties may be complexed to sugars and proteins in a cell membrane. The vast majority of mitochondrial membranes are composed of glycerophospholipids [20], which contain a glycerol backbone, a hydrophilic head group and fatty acid chains (Figure 1A). Naturally occurring fatty acids typically contain 4 to 28 aliphatic carbons of variable length and saturation: saturates contain no carbon double bonds, monounsaturates contain one double bond and polyunsaturates more than one. Figure $1 \mathrm{~B}$ illustrates fatty acid structure and nomenclature.

Extensive work by Daum and colleagues [21,22] has shown that the mitochondrial inner membrane is composed of all the major classes of membrane phospholipids, including phosphatidylcholine, phosphatidylethanolamine, phosphatidylinositol, phosphatidylserine, phosphatidic acid, phosphatidylglycerol and cardiolipin (CL) [22] (Table 1). Mitochondria contain a few other membrane lipids such as sphingolipids and sterols [23], the notable exception being mitochondria involved in steroid synthesis [24].

The different classes of phospholipids and fatty acids confer different properties on the membrane including its ultrastructure. As shown in Figure 2, when the diameters of the hydrophilic head groups and fatty acid chains are similar, the phospholipid molecules take on a cylindrical shape that renders the molecule suitable for forming lipid bilayers. However, small hydrophilic head groups combined with large hydrophobic fatty acid chain diameters lead to a conical shape. This favours a negative curvature, which in vitro forms hexagonal phase structures, but which in vivo is likely to store curvature stress resulting in packing defects and differential lateral pressure profiles, which may affect protein function (reviewed in [25]). Curvature stress energy can affect the binding of membrane proteins within the lipid bilayer or supply energy for protein conformational changes [25]. This is particularly important for the mitochondrial phospholipid CL, whose role in metabolism and lifespan is reviewed later.

Membrane phospholipid and fatty acid compositions are influenced by diet, which can alter membrane composition by several per cent [26]. However, there are much larger species- and tissue-specific differences in fatty acid composition, suggesting an overriding and larger effect of gene expression [15]. Indeed, the fatty acid composition of mitochondrial phospholipids varies widely across species [20] and correlates with body size, basal metabolic rate and longevity [27-29]. 
A

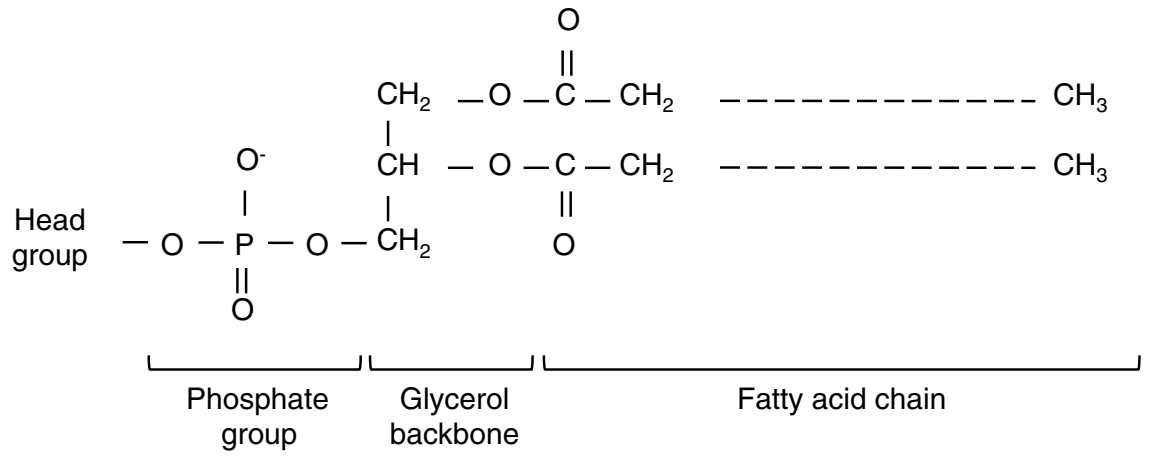

B Linoleic acid (18:2n-6)<smiles>CCCCC/C=C\C/C=C\CCCCCCCC(=O)O</smiles>

Docosahexaenoic acid (22:6n-3)

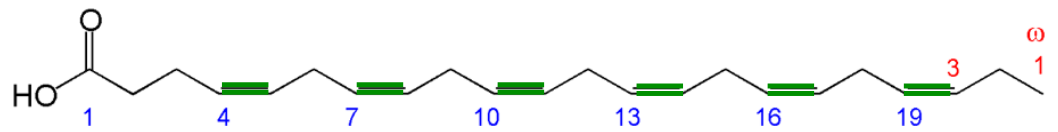

Figure 1 Glycerophospholipids and fatty acids in mitochondrial membranes. (A) Phospholipid molecules are composed of a glycerol backbone to which are attached (sn-1 and sn-2 hydroxyl groups) fatty acid chains of variable length and number of double bonds. A hydrophilic head group can be attached via a phosphodiester bond formed at the sn-3 position. (B) The first carbon in carboxylic acids is at the alpha end. The last carbon is at the omega ( $n$ ) end. For omega-3 ( $n-3)$ fatty acids, such as docosahexaenoic acid, the first double bond is three carbons from the omega end. The first double bond for omega-6 (n-6) fatty acids, such as linoleic acid, is at the sixth carbon from the omega end. Examples of nomenclature are shown: the carbon chain length beginning from the alpha end is shown in blue. The carbon double bonds are shown in green. The position of the first carbon double bond from the omega end is shown in red.

\section{Does mitochondrial membrane composition affect metabolic rate and longevity?}

An allometric comparison of metabolism between ectotherms and endotherms indicates that longer-lived slower metabolizing ectotherms such as lizards also have very low levels of membrane polyunsaturated fatty acids. By contrast shorter-lived endotherms, such as mice and rats, with higher metabolic rates, have highly unsaturated membranes [11]. This observation forms the basis for the 'membrane pacemaker hypothesis of metabolism', which posits various hypotheses to explain how membrane fatty acid composition may causally affect basal metabolic rate, and by extension longevity. It may do so by altering the function of embedded proteins [30] or by

Table 1 Lipid composition of mitochondrial outer (MOM) and inner (MIM) membranes in mammals, plants and yeast ${ }^{\mathrm{a}}$

\begin{tabular}{|c|c|c|c|c|c|c|}
\hline & \multicolumn{2}{|c|}{$\begin{array}{l}\text { Mammalian cells } \\
\text { (Rat liver) }\end{array}$} & \multicolumn{2}{|c|}{$\begin{array}{c}\text { Plant cells } \\
\text { (Cauliflower) }\end{array}$} & \multicolumn{2}{|c|}{$\begin{array}{c}\text { Yeast } \\
\text { (Saccharomyces cerevisiae) }\end{array}$} \\
\hline & MOM & MIM & MOM & MIM & MOM & MIM \\
\hline \multirow[t]{2}{*}{ Phospholipid (mg/mg protein) } & 0.45 & 0.2 & 0.63 & 0.41 & 0.91 & 0.15 \\
\hline & \multicolumn{6}{|c|}{ Percentage of phospholipid } \\
\hline Phosphatidylcholine & 54 & 40 & 47 & 42 & 46 & 38 \\
\hline Phosphatidylethanolamine & 29 & 34 & 27 & 38 & 33 & 24 \\
\hline Phosphatidylinositol & 13 & 5 & 23 & 5 & 10 & 16 \\
\hline Phosphatidylserine & 2 & 3 & - & - & 1 & 4 \\
\hline Cardiolipin & $<1$ & 18 & 3 & 15 & 6 & 16 \\
\hline Phosphatidic acid & 1 & - & - & - & 4 & 2 \\
\hline
\end{tabular}

${ }^{a}$ Mitochondrial phospholipid levels were measured relative to mitochondrial protein levels and the individual phospholipid classes were calculated as a percentage of the total. Data from [22]. 


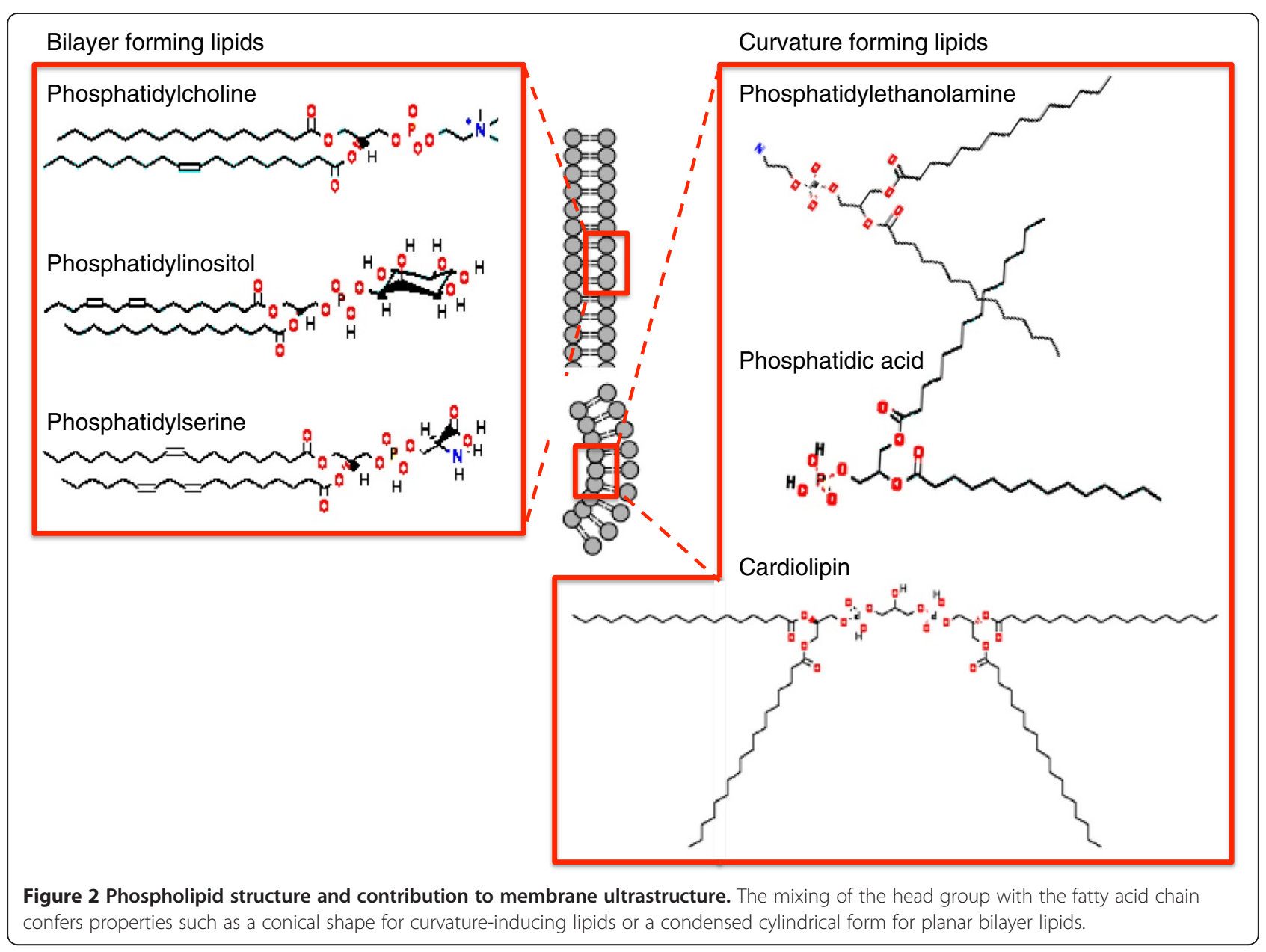

changing permeability and/or the proton leak across the inner mitochondrial membrane [10].

Several studies have shown that changes in membrane fatty acid composition affect protein function in mitochondria, for proteins such as succinate dehydrogenase [31] and cytochrome c oxidase [32], as well as other membrane proteins such as the $\mathrm{Na} / \mathrm{K}$ antiporter [30], which accounts for $10 \%$ to $60 \%$ of the resting metabolic rate according to tissue type $[33,34]$. In particular, $\mathrm{Wu}$, Else and Hulbert conducted some elegant endotherm/ ectotherm crossover studies [30] to show that membrane composition and fatty acid packing in monolayers affects $\mathrm{Na} / \mathrm{K}$ antiporter activity [35] and thereby metabolic rate. Whilst these studies may explain why membrane composition is linked to basal metabolism, they do not support the view that altering the function of embedded membrane proteins might affect lifespan and aging, and we are not aware of any other studies showing such a correlation. Furthermore, we suggest that empirical comparisons of membrane parameters for phylogenetically distant groups such as ectotherms and endotherms are complicated by differences in temperature regulation and weight-specific metabolism, which should be corrected for where possible.

\section{Mitochondrial membrane composition affects proton leak and metabolic rate but is not associated with longevity}

Figure 3 illustrates the coupling between substrate oxidation and ejection of protons by the electron transport chain from the matrix side to the intermembrane space, thus generating a protonmotive force [36]. This electrochemical gradient can then be used to drive energy (ATP) production through ATP synthase [37]. However, electrochemical transduction is not perfectly coupled [38] and protons can leak back from the intermembrane space to the matrix via various processes including passive gradient-dependent cycling carried out by membrane fatty acids or directly by activation of proteins such as the mitochondrial uncoupling proteins (UCPs) [39]. This is termed proton leak, or uncoupling.

Brookes and colleagues [40] have shown that in simplified liposome systems from the phospholipids of eight vertebrates, representing a tenfold range of mitochondrial proton leak and a threefold difference in membrane 


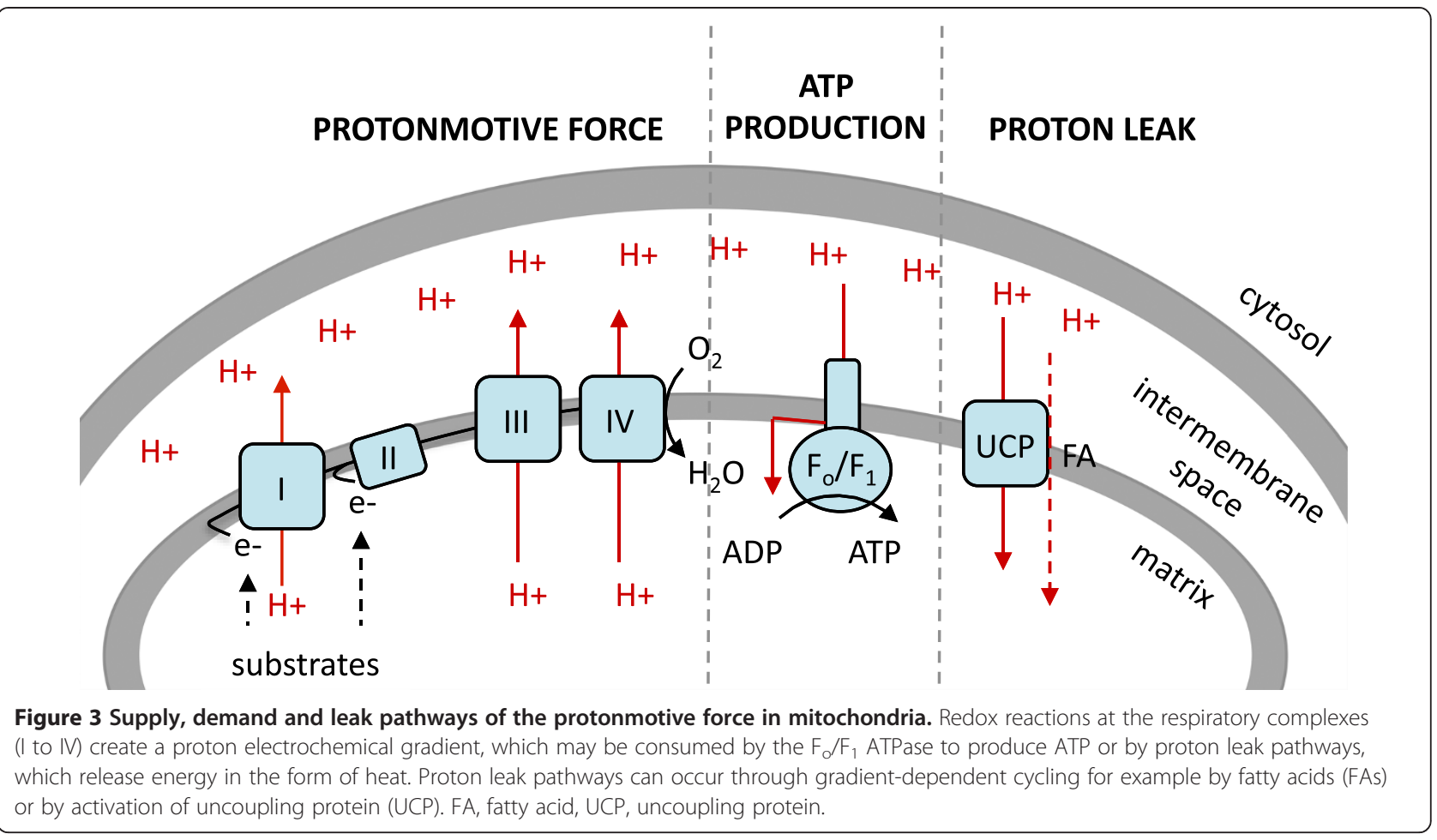

unsaturation, the mitochondrial proton leak was similar. In a subsequent study on isolated mitochondria [10], they showed that proton leak (per milligram of mitochondrial protein) correlated with increased membrane unsaturation. Conversely, a low proton leak was associated with decreased metabolism and increased monounsaturates in the membrane. Thus, Brookes et al. concluded that mitochondrial fatty acid composition might affect the behaviour of one or more mitochondrial inner membrane proteins and thereby might affect the proton leak [10]. Furthermore, the proton leak via the lipid portion of the mitochondrial inner membrane was estimated to be only $5 \%$ of the total membrane proton leak, again suggesting that fatty acid composition might influence the proton leak via proteins, but was not the primary mediator of the process [41]. There is now good evidence that both fatty acids, especially polyunsaturates [42], and lipid peroxidation products [43] activate uncoupling proteins. The activation of uncoupling proteins by products of reactive oxygen species is thought to act as a negative feedback loop to decrease production of such species [44]. By consuming and lowering the protonmotive force, uncoupling decreases the steady-state concentration of carriers that are likely to donate an electron to oxygen to generate ROS $[39,43]$.

An exceptional finding to the membrane pacemaker hypothesis of metabolism is that of birds, which have a higher metabolic rate and generally live longer than sizematched mammals. One might suppose that this can be explained through mild uncoupling in birds, which would increase metabolic rate, but decrease ROS production, thus potentially explaining their longevity. However, studies have shown conflicting results in proton leak rates [10] or ROS production $[8,16,45]$ in birds compared with size-matched mammals. The question of whether membrane lipids are directly correlative with uncoupling in mediating lifespan extension remains unanswered. Combining studies looking at membrane composition and uncoupling [46,47], and membrane composition and lifespan [14,38] requires unsafe assumptions that result in conflicting outcomes. The topic of uncoupling and lifespan is extensively reviewed elsewhere [48].

\section{Membrane unsaturation and peroxidation}

Studies by the Pamplona and Barja group first suggested that low fatty acid unsaturation in mitochondria protects against lipid peroxidation in liver mitochondria for the long-lived pigeon compared to the shorter-lived but phylogenetically very distant rat [49]. In a subsequent study, they showed that the extent of membrane unsaturation was directly correlated with increased lifespan in several mammals [50], although their data were not specific to mitochondrial membrane composition.

In Table 2, we have compiled the mean mitochondriaspecific content of saturated (SFA), monounsaturated (MUFA), polyunsaturated (PUFA) fatty acids and the highly unsaturated PUFA docosahexaenoic acid (DHA) 
Table 2 Mitochondrial membrane fatty acid composition in a range of tissues from endothermic and exothermic species

\begin{tabular}{|c|c|c|c|c|c|c|c|c|}
\hline Species (common name) & Mass (kg) & MLSP (\%) & SFA (\%) & MUFA (\%) & PUFA (\%) & DHA (\%) & Tissue & Reference \\
\hline Pogona vitticeps & 0.5 & 12 & 21.8 & 29.1 & 49.1 & 0.7 & Liver & {$[52]$} \\
\hline \multirow[t]{2}{*}{ (bearded dragon lizard) } & & & 25.7 & 26.9 & 47.4 & 0.9 & Kidney & \\
\hline & & & 18.6 & 27.9 & 53.5 & 3.3 & SKM & \\
\hline Bufo marinus & 0.1 & 24.8 & 26.3 & 24.7 & 49 & 2.2 & Liver & {$[52]$} \\
\hline \multirow[t]{2}{*}{ (cane toad) } & & & 19 & 25.5 & 55.5 & 1.4 & Kidney & \\
\hline & & & 17.6 & 21.9 & 60.5 & 2.2 & SKM & \\
\hline Columba livia & 0.36 & 35 & 38.5 & 13.2 & 48.3 & 2.1 & Liver & [7] \\
\hline \multirow[t]{2}{*}{ (pigeon) } & & & 43.4 & 7.8 & 48.9 & 1.3 & SKM & \\
\hline & & & 41.1 & 13 & 45.9 & 1.7 & Heart & \\
\hline Coturnix chinensis & 0.05 & 5 & 40.8 & 15.7 & 43.5 & 6.5 & Liver & [7] \\
\hline \multicolumn{9}{|l|}{ (king quail) } \\
\hline Coturnix japonica & 0.12 & 6 & 41.9 & 14.6 & 43.6 & 7.3 & Liver & [7] \\
\hline \multicolumn{9}{|l|}{ (Japanese quail) } \\
\hline Agapornis spp. & 0.05 & 16 & 38.4 & 19.2 & 42.5 & 3.0 & Liver & [7] \\
\hline \multicolumn{9}{|l|}{ (lovebird) } \\
\hline Nymphicus hollandicus & 0.09 & 35 & 42 & 17.5 & 40.5 & 3.8 & Liver & [7] \\
\hline \multicolumn{9}{|l|}{ (cockatiel) } \\
\hline Eutamias amoenus & & & 35.6 & 24.1 & 40.3 & 6.47 & Liver & [53] \\
\hline (yellow pine chipmunk) & & & 37.4 & 12.5 & 50.1 & 27.3 & Heart & \\
\hline \multirow[t]{2}{*}{ Rattus norvegicus } & & & & & & 7.8 & Liver & {$[8,9,52]$} \\
\hline & & & & & & 2.1 & Kidney & \\
\hline \multirow[t]{2}{*}{ (rat) } & 0.35 & 2.8 & & & & 14.9 & SKM & \\
\hline & & & 32.9 & 13 & 54.2 & 7.8 & Heart & \\
\hline Mus musculus, C57BI/6 & 0.02 & 4 & 34 & 11 & 55 & 21.9 & Liver & $* *$ \\
\hline \multicolumn{9}{|l|}{ (house mouse) } \\
\hline Mus musculus, Ames dwarf & 0.01 & 5 & 38.9 & 8.8 & 52.4 & 9.97 & Liver & [54] \\
\hline \multicolumn{9}{|l|}{ mouse (house mouse) } \\
\hline Peromyscus maniculatus & 0.02 & 8.3 & 32.6 & 9.75 & 57.7 & 34.9 & Heart & [55] \\
\hline
\end{tabular}

DHA docosahexaenoic acid; MLSP, maximum lifespan; MUFA, monounsaturated fatty acid; PUFA, polyunsaturated fatty acid; SFA, saturated fatty acid; **, TGV, unpublished data. SKM skeletal muscle.

in different tissues from a range of species including ectotherms, birds and mammals. In addition, we list the species-specific body mass as well as maximum lifespan (MLSP), both obtained from the AnAge database [51]. Whilst levels of mitochondrial SFA, MUFA and PUFA appear to be constant in species with different lifespans, levels of the highly unsaturated lipid DHA, decrease dramatically with increasing lifespan, and this is demonstrated graphically in Figure 4. Using correlative measures only, we find this strong relation for DHA disappears when plotting liver tissue only (not shown) but reappears with plotting heart data (Figure 4D). We acknowledge, however, that the compiled fatty acid profiles from isolated mitochondria in Table 2 and Figure 4 are somehow limited and contain information from very distinct taxa and with large within-rodent taxon clustering. For this reason, we have not conducted statistical analyses with corrections for body mass and phylogeny as this is likely to produce erroneous results. Further work will be required to amass sufficient data from many different species to conduct large-scale analyses.

The mechanism linking mitochondrial membrane unsaturation and aging might be as follows. Mitochondrial free radicals generated as a product of the respiratory chain during oxidative phosphorylation initiate the formation of a lipid radical. The presence of a methylene bridge adjacent to a carbon double bond is particularly susceptible to attack by oxygen free radicals and can 
A

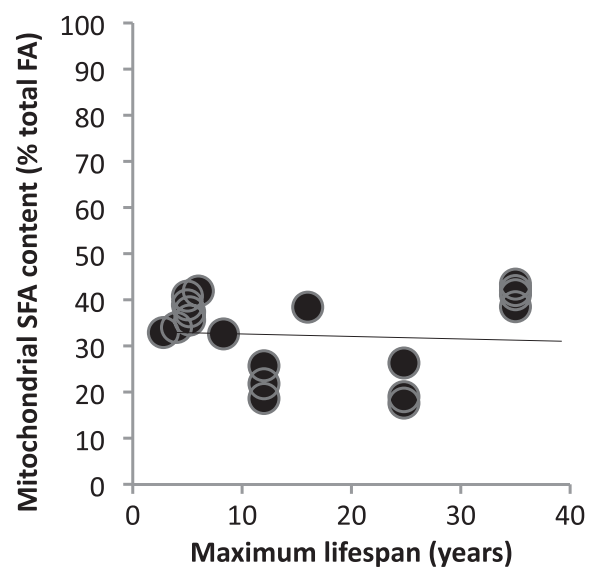

C

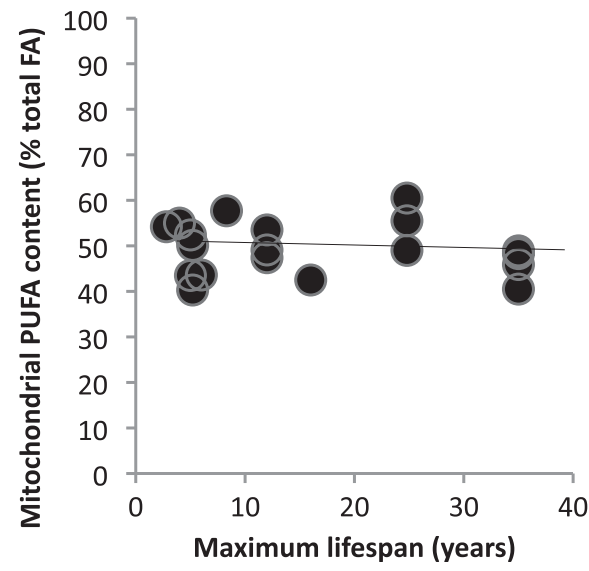

B

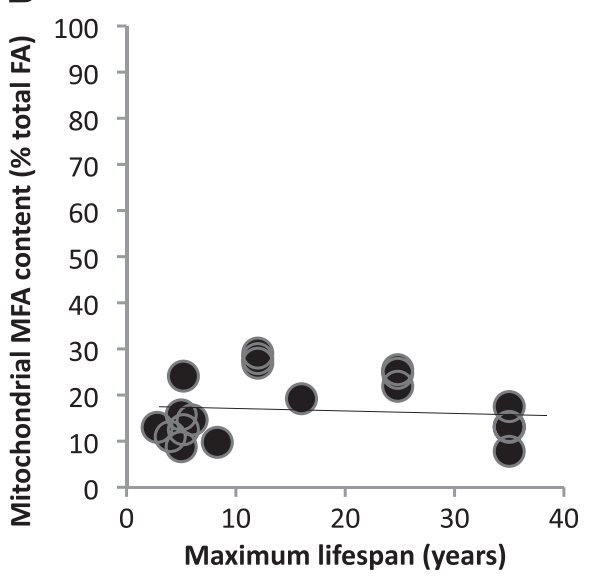

D

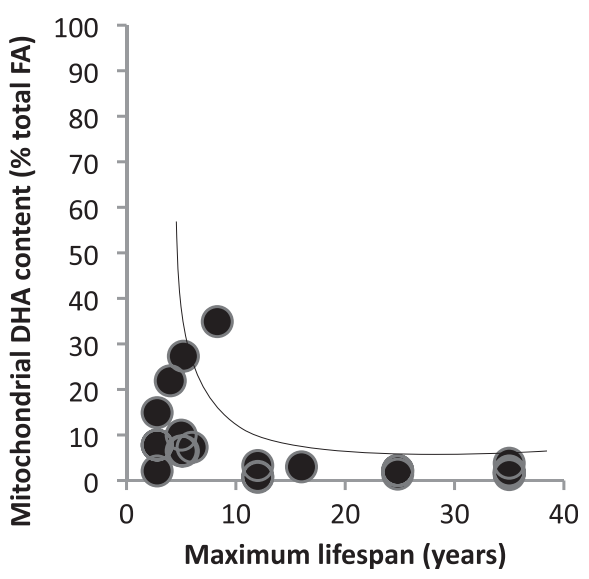

Figure 4 Fatty acid contents for a range of tissues from endothermic and exothermic species. (A) Mitochondrial saturated fatty acids. (B) Monounsaturated fatty acid. (C) Polyunsaturated fatty acids. (D) Docosahexaenoic acid. The data are graphical displays of the fatty acid content detailed in Table 2. These data have not undergone statistical analysis, as described in the main text. DHA, docosahexaenoic acid; FA, fatty acid; MUFA, monounsaturated fatty acid; PUFA, polyunsaturated fatty acid; SFA, saturated fatty acid.

form carbon-centred radicals with subsequent propagation of peroxyl radicals. These lipid peroxidation products result in membrane degeneration as well as protein and genome toxicity [56], culminating in aging and death (Figure 5).

Such observations $[27,49,50]$, led Pamplona and Barja to propose the homeoviscous-longevity adaptation hypothesis: namely, that the lower degree of fatty acid unsaturation in longevous animals decreases their sensitivity to lipid peroxidation and macromolecular damage. They have since published two indices, which they suggest causatively correlate with lifespan: the double bond index [13], later refined to the peroxidation index (PI) [57]. Both indices describe the risk of oxidation of unsaturated fats, the explanation again being that lower PI leads to less lipid and downstream protein and DNA oxidative damage and therefore leads to lifespan extension. Valencak has recently independently found that for isolated mitochondria of long-lived Ames dwarf mice, their PI was 13\% lower than that of their heterozygous short-lived siblings [54].

However, we wish to point out several caveats with using PI to explain aging and longevity causally. Firstly, PI does not take into account that saturated fatty acids can under certain conditions be more susceptible to oxidation than unsaturated fatty acids, especially some shorter chained fats [58]. Secondly, PI largely reflects the content of one very highly unsaturated PUFA, DHA. Indeed, DHA is really the predominant PUFA that has been linked with MLSP $[14,27,49]$ irrespective of total PUFA levels, which appear not to vary with lifespan [14] (Figure 4). Thirdly, molecules other than lipid peroxides may ultimately be more important in mediating cellular oxidative damage. For example, research in humans and animal models reveals that the aldehydic lipid peroxidation products, 4-hydroxyl-2-nonenal and malonaldehyde (MDA), are more stable than lipid peroxyl radicals and 


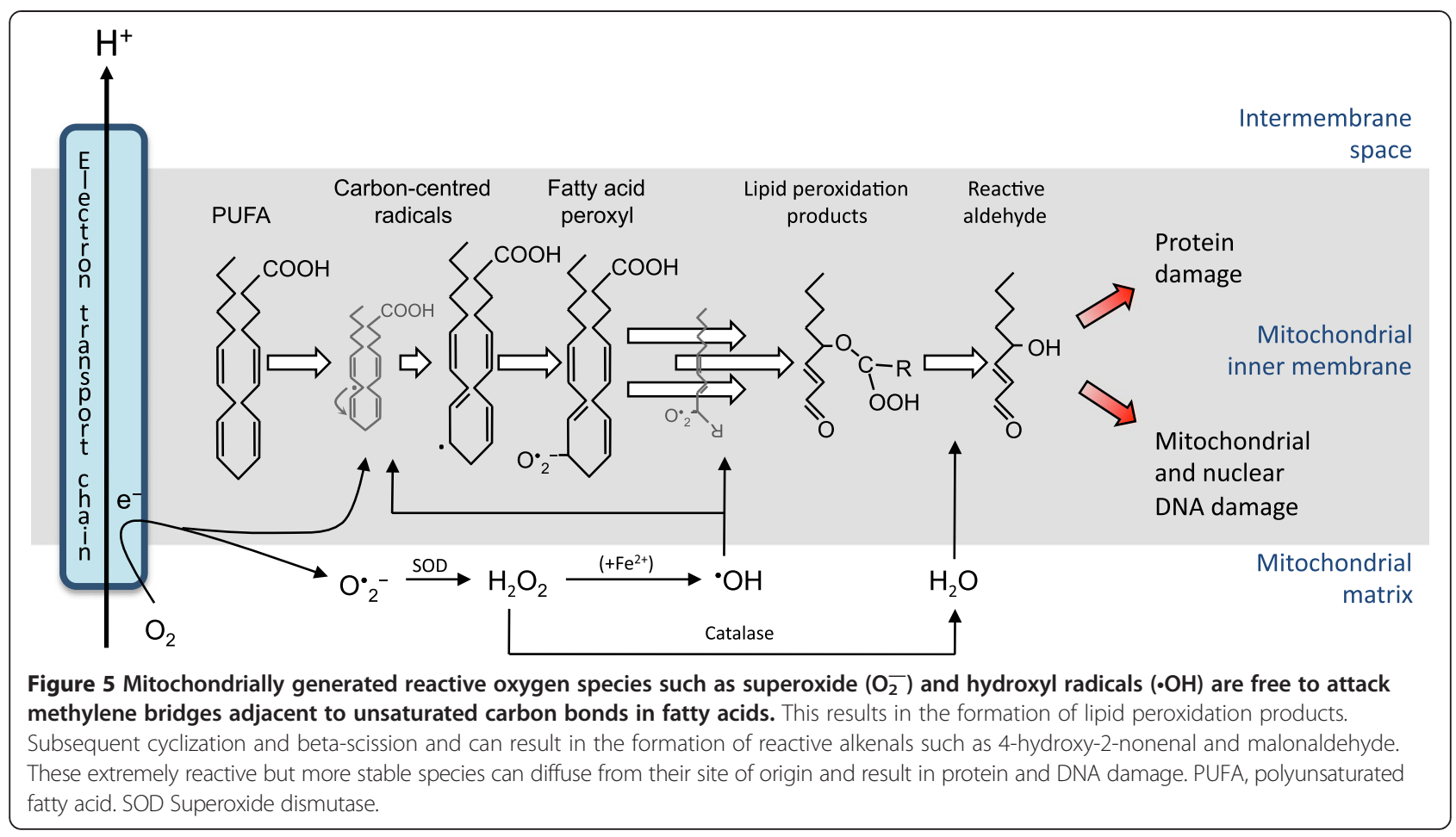

are therefore able to diffuse from their origin to induce greater cellular damage [59].

Interestingly, Montgomery and colleagues recently reported no difference in the fatty acid composition (including $\mathrm{n}-3$ fatty acids) or PI between the long-lived cockatiel (MLSP 35 years) and short-lived quails (MLSP 5.5 years) [7]. Although the animals studied were all aged one regardless of lifespan difference, this suggests at the very least that either membrane composition bears no relation to lifespan, or that membrane composition at a relatively early age does not predict longevity.

An alternative suggestion to the homeoviscous-longevity adaptation hypothesis might be that if lipid peroxides can act as ROS scavengers [60] rather than simply propagators of ROS as traditionally thought, then possibly the higher PUFA levels in shorter-lived animals may simply reflect a physiological adaptation to a stressful milieu.

\section{Oxidative stress in the naked mole rat}

Buffenstein and colleagues, approached the question as to whether damage generation underlies species longevity by comparing oxidative damage in a long-lived rodent, the naked mole rat (MLSP >28 years) with the comparably sized mouse (MLSP 3.5 years) [61-63]. Surprisingly and contradicting the oxidative stress hypothesis of aging, concentrations of markers of DNA damage and lipid peroxidation were greater in naked mole rats than in mice, even at a young age [62]. This is in line with data that show greater hydrogen peroxide production than expected from naked mole rat mitochondria [64]. Furthermore, contrary to predictions that oxidative stress increases with aging within species, lipid damage levels did not change with age in naked mole rats [62].

Interestingly, naked mole rats do have a membrane composition that fits with the aforementioned theoretical predictions about lifespan [65]. Compared with mice, naked mole rats have one-ninth the content of highly unsaturated DHA, despite maintaining the same overall phospholipid content [65]. Mitchell and colleagues [65] postulate that this lowers their susceptibility to peroxidative damage and state that the original findings for higher levels of lipid peroxides were because the urinary isoprostanes and liver malonaldehyde (MDA) measured in the Andziak study [62] were specific products of arachidonic acid (C20:4n-6) but not of the more unsaturated DHA (C22:6n-3) [62,65]. Furthermore, Mitchell et al. found increased plasmenyl lipid levels for the longer-lived naked mole rats compared to mice [65], and postulated, based on previous studies, that they may act as membrane antioxidants [66,67], so explaining the longer lifespan in these species.

However, whilst we would agree that urinary isoprostanes are products of esterified arachidonic acid, MDA is a known product of both arachidonic acid and DHA [59] and reasonably reflects lipid damage in naked mole rats. Additionally, Mitchell and colleagues do not attempt to explain why, if reduced DHA and increased levels of plasmenyl lipids in mole rats provide a protective mechanism 
against oxidative damage, these animals have increased mitochondrial and nuclear DNA damage as well as increased MDA levels.

Interestingly, the Mitchell study used assumptions based on previous work, which showed that only four fatty acid species are de novo synthesized whilst the rest are remodelled by enzymatic deacylation-reacylation [68]. They demonstrated that for naked mole rats compared to mice, the relative balance of fatty acids is shifted away from de novo synthesis and towards remodelling [65]. Supposing the assumptions apply correctly, this may reflect a system compensation for high oxidative stress levels, just as Andziak's work has demonstrated that peroxiredoxin (an important antioxidant) in naked mole rats may suffer high levels of damage in keeping with its specific function [61]. Similarly, increased levels of plasmenyl lipids [65] may be a compensatory mechanism for high oxidative stress rather than a causative link with longevity. Correlations between levels of plasmenyl lipids and lifespan have not been investigated elsewhere and it would be interesting to conduct this work for a broader range of species.

\section{Unsaturation in hibernators}

The extent to which dietary PUFAs influence mitochondrial membrane phospholipids was first addressed for deer mice [55], chipmunks [53] and golden-mantled ground squirrels [69]. These studies were designed to identify the role of dietary PUFAs on torpor patterns and hibernation, and revealed that dietary PUFAs (for example, supplementary C18:2n-6 or C18:3n-3) led to a $7 \%$ increase in mitochondrial PUFA content and that these changes were paralleled by a $2.5^{\circ} \mathrm{C}$ decrease in minimum body temperature and longer torpor bouts [70,71]. The duration and extent of hypothermic phases were improved by PUFAs through establishing and maintaining high membrane fluidity [72] and lowering enzyme activity, for example, for cytochrome c oxidase [73]. In other words, increased levels of PUFAs allowed for slowed metabolism and reportedly, had very beneficial effects on the survival of the animals [74].

At the time, however, scientists largely overlooked the predictions from the membrane pacemaker hypothesis of metabolism and aging and left unnoticed the fact that membrane unsaturation or PUFA contents in membranes of different tissues consistently increase in all species observed when an animal becomes torpid and lowers its metabolism (cf the membrane pacemaker hypothesis of metabolism). It took two more decades before Gerson et al. [75] compared mitochondrial metabolism between torpid and euthermic 13-lined ground squirrels. They observed that during hibernation, respiration and proton leak were suppressed as expected [75]. Unexpectedly given the lower respiration, membrane unsaturation increased while the animal was torpid and lipid peroxidative damage increased twofold as assessed by MDA levels in isolated liver mitochondria [75]. Although in a subsequent study by the same group and using the same species, mitochondrial ROS production appeared to decrease during hibernation, the assay used in that study detected hydrogen peroxide in the cytosol rather than other free radicals produced intra-mitochondrially [76]. Thus, while the pattern of increasing membrane unsaturation in the course of hibernation is consistent [77], lipid peroxidation during hibernation still remains a matter of debate.

\section{Cardiolipin: the mitochondrial phospholipid}

Unlike other membrane lipids, CL is a dimerically crosslinked phospholipid that, in eukaryotes, is found almost exclusively in mitochondria and almost entirely in their inner membrane [22] (Table 1). This makes it interesting to investigate in terms of the link between mitochondrial membranes and longevity.

Because of its unique dimeric structure, CL has two glycerol backbones each with a chiral centre and four fatty acid chains, making the potential for complexity rather large (Figure 2). In eukaryotic tissues ranging from fungi to mammals, CLs contain mainly monounsaturated or di-unsaturated chains with 16 or 18 carbon atoms. This restricted fatty acid chain length and saturation result in a relatively homogeneous distribution of double bonds and carbon numbers among the four acyl chains [78].

In the mitochondrial inner membrane, $\mathrm{CL}$ is involved in stabilising membrane proteins including respiratory complexes [79] and the adenine nucleotide transferase [80]. Furthermore, studies show that CL directly influences the function of the adenine nucleotide transferase [81], an important mitochondrial enzyme that allows the import of ADP into mitochondria for ATP synthesis, and ejects synthesized ATP for use in intracellular processes. In the mitochondrial outer membrane, CL has been suggested to be present in and to be implicated in the function of the protein import machinery of mitochondria (reviewed in [82]). It has also been shown to have a role in regulating apoptosis through several mechanisms including interaction with caspase 8 [83] and cytochrome c [84], as well as playing a vital role in mitochondrial network morphology through interaction with fission/fusion proteins in the outer membrane (reviewed in [82]).

Despite CL's physiological importance and its partial susceptibility to oxidative damage due to the presence of four unsaturated fatty acid chains, there is weak evidence that CL itself impairs or promotes longevity.

Many studies have used methodological approaches that provide mechanistic insights and possibly allow the authors to comment on CL's putative role in 'aging' but 
not in lifespan [85]. For example, the response of young and aged mitochondria to exogenously supplemented CL cannot address the role of CL in lifespan [86].

At best, one yeast study showed that impaired CL synthesis lead to decreased longevity, which was restored by enhancing the stress response pathways and promoting cellular integrity using an osmotic stabiliser [87]. Although certain studies showed decreased CL levels in aged worms [88], this was consistent with their finding of decreased mitochondrial numbers and hence membranes. Interestingly, for aged rats, there is some evidence that CL fatty acid chains are remodelled from linoleic acid (18:2n-6) to the more unsaturated arachidonic (20:4n-6) and docosahexaenoic (22:6n-3) acids [89]. There is evidence elsewhere that remodelling occurs in other phospholipid species. In pulse-label experiments of phosphatidylcholine and phosphatidylethanolamine, Schmid et al. showed that only four fatty acid species were de novo synthesized (6:0-18:2 (n-6), 16:0-18:1, 16:0-22:6 (n-3) and 18:1-18:2(n-6)), whilst the remainder were remodelled via rapid deacylation-reacylation [68]. This may explain why in a recent phylogenomic study by Jobson [90] examining codon evolution across 25 mammalian species with different longevities, of genes with significantly high evolutionary selection in long-lived species there were a number of lipid membrane composition genes. These were fatty acid elongases, desaturases and fatty acid synthases including those involved in the reconstruction of membrane CLs [90]. Again, these studies may echo our previous suggestion than PUFA levels are a response to cellular stress rather than being a causative agent in aging.

\section{Phylogenetic and allometric corrections: a beautiful theory killed by an ugly fact?}

Simple correlations between the phospholipid composition of mitochondrial membranes, metabolism and longevity bear two notable risks. Firstly, fatty acid composition, as probably all other physiological traits, correlates with body mass because body mass represents a most 'pervasive trait influencing all aspects of organismal biology' [91]. By simply relating the DHA content in a given membrane or tissue to maximum lifespan, one might end up having a close correlation between the two but the traits might actually have no functional relation to each other. For example, DHA might be simply more enriched in mitochondrial membranes of a mouse compared to an elephant due to allometry [15]. Secondly, although independent replicates are prerequisites for applying powerful parametric statistical tests, both Speakman $[16,91]$ and Valencak and Ruf [15] point out that different species do not represent independent replicates as they may be phylogenetically correlated despite not sharing the same ancestor.
To overcome both of the above issues, statistical 'remedies' that simultaneously correct for body weight and phylogeny have been developed and are freely available online in the form of multivariate regression analysis and the package APE in R [92]. Additionally, this area of research has greatly benefitted from advances in genetics and DNA sequencing, so permitting the accrual of more accurate phylogenetic relationships among species.

Previously, Valencak has found that applying this corrective statistical approach to a large dataset of mammals (using the package Phylogr in R), several of the reported relations fell apart for example, between DHA and MLSP [15]. Similar corrections linking other traits, such as ROS production, with lifespan in a comparative dataset have seen a similar loss of statistical significance once body mass and phylogenetic corrections were made [64]. Therefore, as well as suggesting the re-examination of previous work conducted without accounting for body weight or phylogeny, we recommend that future comparative studies should employ the suggested methodology to allow for better correlation of physiological traits with longevity.

It has been argued by critics of this approach that correcting for body weight and phylogeny might be overly conservative, as it may wipe away important variation in the data that co-explains the observed relations. However, from an evolutionary and comparative physiological perspective, especially in light of improved statistical approaches compared with the older residual analysis approach [93], we suggest the use of body weight- and phylogeny-corrected statistical approaches for all comparative datasets, especially those relating to aging and lifespan. While statistically eliminating the influence of body size might mainly affect the interpretation of comparative datasets, we even previously suggested that, given a large sample with little within-taxon clustering, the incorporation of phylogeny into the models may not affect the interpretation of the main findings but instead give results at a much finer resolution [15]. Undoubtedly, the correction for body weight with the resulting lack of a relation clearly indicates that many of the so-far reported correlations might in fact have been 'spurious' [91] or, at least, the magnitude of the association might be much smaller than suggested by simple cross-species correlations.

\section{Caloric restriction and fatty acid metabolism: all about the omega?}

Valencak and Ruf's use of statistical models that adjust for body weight and phylogeny showed that in contrast to previous studies, there was no relation between MLSP and membrane unsaturation, DHA content or peroxidation index [15]. Only one parameter correlated significantly with lifespan: the ratio of n-3:n-6 PUFAs, with decreased 
with increasing lifespan. These findings mimic the wellknown difference in the n-3:n-6 PUFA ratio between mammals and birds of similar size: the relatively longevous birds have lower n-3:n-6 PUFA ratios [12].

Interestingly, caloric restriction without malnutrition the only effective physiological means of extending lifespan for a large range of species [94] - results in a decrease in the percentage of $n-3$ and an increase in n-6 PUFAs [95]. By using Weindruch's paradigm and calorically restricting mice at different levels, Faulks et al. noted a clear decrease in n-3 PUFAs and in the n-3:n-6 ratio in mitochondrial phospholipids from skeletal muscle, liver, brain and heart tissues [96]. Of note, they did not find pronounced differences in ROS production in the animals [96]. However, a more recent study by Valencak shows that despite changes in $n 3: n 6$ PUFA ratios (and PI) in mice fed different diets, there was no difference in longevity [26]. From a physiological viewpoint, the significant relation between the n-3:n-6 PUFA ratios and MLSP might be linked to some other feature rather than being causative for senescence and aging.

The literature suggests that the general biochemical and physiological observations for the link between membrane composition and lifespan appear to be supported by genomic studies. This includes a phylogenomic study by Jobson et al. showing increased evolutionary selective pressure for genes encoding membrane composition in longer-lived mammals [90] and gene ablation studies of membrane composition genes [97].

However, closer inspection raises uncertainties. The Jobson study [90] does not reflect that higher animals are unable to de novo synthesize n-3 and n-6 PUFAs, thus explaining why they might exert a stronger evolutionary selection on elongase and desaturase enzymes. To illustrate this point another way, their work showed that genes ensuring genome integrity did not have a strong selective pressure in longevous animals compared to shorter-lived ones. This is unsurprising given that all animals probably need a similar level of strong control over their genome to prevent cellular and organismal death; but this is not evidence that genome integrity is not important in longevity. Certainly their findings are novel, interesting and require further investigation, including correcting for body mass and investigating species across different taxonomic orders, which are likely to have different selective pressures.

Other studies investigating the effect of ablation of membrane composition pathway genes on lifespan extension are likely to be of huge importance in shedding mechanistic light on the topic; however, contemporary studies suffer from using the same oxidative stress hypotheses to explain lifespan extension without actually measuring oxidative damage species [97].
So while it appears that there is reasonable circumstantial evidence for a link between membrane composition and longevity, there is no evidence of causation. More mechanistic work and a range of species will be needed to decipher how and why membrane composition might correlate with lifespan and whether it is causative.

We suggest that for future studies, the following points ought to be addressed:

- The use of too small a comparative sample, that is, too few species from overly close taxonomic groups. Optimal comparative datasets should contain samples from whichever and as many species as can be sampled.

- The use of potentially inappropriate measures, for example, basal metabolic rate (which represents the minimum energy requirement for staying alive) as opposed to average daily energy use [16]; or using maximum lifespan (clearly an extreme characteristic) rather than the median of an upper percentile of longevous years. Although this issue is contentious, it merits discussion and attention [91].

- Statistical analysis without attention to confounding covariates, for example, fatty acid composition and correlation with lifespan without correction for body mass or phylogeny. Phylogenetic correction is particularly important for comparative studies containing within-taxon clustering where statistical degrees of freedom would be clearly exaggerated if used as independent data replicates.

- Correlation being confused with causation without sufficient evidence or logical premise, or without due attention to confounding mechanisms, for example, polyunsaturated lipid peroxides causing aging rather than being associated with it for some other reason including physiological responses to stress.

\section{Conclusions}

The concept of immortality and longevity has probably captivated humankind from the earliest days and was first recorded by the Babylonians 4,000 years ago. Yet, only in the last two centuries have we made some progress in attempting to answer the question: 'What makes some organisms live longer?' Starting from body mass correlations, through to metabolic rate, oxidative stress and membrane fatty acid composition, the field remains complex and burdened with irregularities.

We conclude from reviewing the literature available on mitochondrial lipid composition that there may well be an association between high levels of membrane n-3 PUFAs and a comparatively low longevity, as would be predicted by the homeoviscous-longevity adaptation or 
the membrane pacemaker hypotheses of aging. However, our overview also suggests that the magnitude of this association might vary according to the tissues and datasets used (such as the specific animal taxa or even knock-out mouse models). It is currently difficult to appreciate whether and which tissues are important in correlations between membrane composition and longevity. Likewise, the extent of the relation between membrane composition and MLSP might have been overestimated in the past due to various reasons, including the lack of powerful statistical approaches built on reliable phylogenetic information. Comparative datasets that do not correct for phylogeny or co-variation of traits with body mass may easily lead to oversimplified relations for certain physiological traits and maximum lifespan. The statistical approaches developed by comparative biologists may be powerful tools for getting new and more accurate information out of comparative differences in the aging process across phylogenetically very different species.

On a side note, we observe a difference in the perception as to which fatty acid classes are beneficial or detrimental to human health span. Among comparative physiologists, saturated fatty acids are considered rather harmless constituents of membranes and tissues. They have a fixed amount in membranes and bring about little if any cellular damage due to peroxidation. In contrast, nutritionists and physicians perceive that saturated fats bring about an increased risk of cardiovascular disease. Abbott and colleagues recently showed that extensive changes in the SFA, MUFA and PUFA levels of diets had minimal effects on the fatty acid composition of membranes in rats but considerable influence on adipose tissue and plasma triglycerides [98]. This likely explains the difference in perception and fits in with epidemiological and clinical evidence suggesting that mortality due to coronary heart disease can be reduced by partly replacing dietary saturated fats with polyunsaturated fats while maintaining a low intake of trans fatty acids $[99,100]$. This dietary modification would reduce serum concentrations of triglycerides and cholesterol, which is a well-established risk factor for heart disease [101].

\section{Abbreviations \\ CL: cardiolipin; DHA: docosahexaenoic acid; FA: fatty acid; MDA: malonaldehyde; MIM: mitochondrial inner membrane; MLSP: maximum lifespan; MOM: mitochondrial outer membrane; MUFA: monounsaturated fatty acid; PI: peroxidation index; PUFA: polyunsaturated fatty acid; ROS: reactive oxygen species; SFA: saturated fatty acid; UCP: uncoupling protein.}

\section{Competing interests}

The authors declare that they have no competing interests.

\section{Authors' contributions}

TGV compiled the data on fatty acid composition in different tissues and animals and VA produced the figures and tables. Both authors contributed equally to the conception, evaluation and writing of the manuscript. Both authors gave final approval to the manuscript.

\section{Acknowledgements}

TGV is funded by an Elise Richter fellowship from the Austrian Science Foundation (FWF, V197-B17) and by a fellowship for visiting international scientists from the Chinese Academy of Sciences in China (grant code 2011Y2SA09). We thank Dr. T Schulz, Dr. ER Price and Dr. LME Kennard as well as two anonymous reviewers for helpful comments on the manuscript.

\section{Author details}

${ }^{1}$ Research Institute of Wildlife Ecology, Veterinary University Vienna, Savoyenstrasse 1, 1160 Vienna, Austria. ${ }^{2}$ Institute of Genetics and Developmental Biology, State Key Laboratory of Molecular Developmental Biology, Chinese Academy of Sciences, Beichen Xi Lu, Chaoyang, Beijing, China. ${ }^{3}$ Christ's College \& Department of Medicine, University of Cambridge, Cambridge CB2 3BU, UK.

Received: 28 October 2013 Accepted: 29 January 2014

Published: 3 March 2014

\section{References}

1. Rubner M: Das Problem der Lebensdauer und seiner Beziehungen zu Wachstum und Ernährung. Munich: Oldenburg; 1908.

2. Kleiber M: Body size and metabolism. Hilgardia 1932, 6:315-351.

3. Pearl R: The Rate of Living, Being an Account of Some Experimental Studies on the Biology of Life Duration. New York: Alfred A. Knopf; 1928.

4. Gerschman R, Gilbert DL, Nye SW, Dwyer P, Fenn WO: Oxygen poisoning and x-irradiation: a mechanism in common. Science 1954, 119:623-626.

5. Harman D: Aging: a theory based on free radical and radiation chemistry. J Gerontol 1956, 11:298-300.

6. Metchnikoff E: The Prolongation of Life; Optimistic Studies. GP Putnam's Sons: New York, London; 1908.

7. Montgomery MK, Hulbert AJ, Buttemer WA: Metabolic rate and membrane fatty acid composition in birds: a comparison between long-living parrots and short-living fowl. J Comp Physiol B 2012, 182:127-137.

8. Montgomery MK, Hulbert AJ, Buttemer WA: The long life of birds: the rat-pigeon comparison revisited. PLoS One 2011, 6:e24138.

9. Brand MD, Couture P, Else PL, Withers KW, Hulbert AJ: Evolution of energy metabolism. Proton permeability of the inner membrane of liver mitochondria is greater in a mammal than in a reptile. Biochem J 1991, 275(Pt 1):81-86.

10. Brookes PS, Buckingham JA, Tenreiro AM, Hulbert AJ, Brand MD: The proton permeability of the inner membrane of liver mitochondria from ectothermic and endothermic vertebrates and from obese rats: correlations with standard metabolic rate and phospholipid fatty acid composition. Comp Biochem Physiol B Biochem Mol Biol 1998, 119:325-334.

11. Hulbert AJ, Else PL: Membranes as possible pacemakers of metabolism. J Theor Biol 1999, 199:257-274.

12. Hulbert AJ: Life, death and membrane bilayers. J Exp Biol 2003, 206:2303-2311.

13. Pamplona R, Barja G, Portero-Otin M: Membrane fatty acid unsaturation, protection against oxidative stress, and maximum life span: a homeoviscous-longevity adaptation? Ann N Y Acad Sci 2002, 959:475-490.

14. Hulbert AJ: On the importance of fatty acid composition of membranes for aging. $J$ Theor Biol 2005, 234:277-288.

15. Valencak TG, Ruf T: N-3 polyunsaturated fatty acids impair lifespan but have no role for metabolism. Aging Cell 2007, 6:15-25.

16. Speakman JR: Body size, energy metabolism and lifespan. J Exp Bio/ 2005, 208:1717-1730

17. Kowaltowski AJ: Alternative mitochondrial functions in cell physiopathology: beyond ATP production. Braz J Med Biol Res 2000, 33:241-250.

18. Lanza IR, Nair KS: Mitochondrial function as a determinant of life span. Pflugers Arch 2010, 459:277-289.

19. Barja $G$, Herrero A: Oxidative damage to mitochondrial DNA is inversely related to maximum life span in the heart and brain of mammals. FASEB J 2000, 14:312-318.

20. Daum G: Lipids of mitochondria. Biochim Biophys Acta 1985, 822:1-42.

21. Daum G, Vance JE: Import of lipids into mitochondria. Prog Lipid Res 1997, 36:103-130.

22. Horvath SE, Daum G: Lipids of mitochondria. Prog Lipid Res 2013, 52:590-614. 
23. Bottema CK, Parks LW: Sterol analysis of the inner and outer mitochondrial membranes in yeast. Lipids 1980, 15:987-992.

24. Petrescu AD, Gallegos AM, Okamura Y, Strauss JF 3rd, Schroeder F: Steroidogenic acute regulatory protein binds cholesterol and modulates mitochondrial membrane sterol domain dynamics. J Biol Chem 2001, 276:36970-36982

25. van den Brink-van der Laan E, Killian JA, de Kruijff B: Nonbilayer lipids affect peripheral and integral membrane proteins via changes in the lateral pressure profile. Biochim Biophys Acta 2004, 1666:275-288.

26. Valencak TG, Ruf T: Feeding into old age: long-term effects of dietary fatty acid supplementation on tissue composition and life span in mice. J Comp Physiol B 2011, 181:289-298.

27. Couture $\mathrm{P}$, Hulbert AJ: Membrane fatty acid composition of tissues is related to body mass of mammals. J Membr Biol 1995, 148:27-39.

28. Porter RK, Brand MD: Cellular oxygen consumption depends on body mass. Am J Physiol 1995, 269:R226-R228.

29. Porter RK, Hulbert AJ, Brand MD: Allometry of mitochondrial proton leak: influence of membrane surface area and fatty acid composition. Am J Physiol 1996, 271:R1550-R1560.

30. Else $\mathrm{PL}, \mathrm{Wu} \mathrm{BJ}$ : What role for membranes in determining the higher sodium pump molecular activity of mammals compared to ectotherms? J Comp Physiol B 1999, 169:296-302.

31. Hazel JR: The effect of temperature acclimation upon succinic dehydrogenase activity from the epaxial muscle of the common goldfish (Carassius auratus L.) - II. Lipid reactivation of the soluble enzyme. Comp Biochem Physiol B 1972, 43:863-882.

32. Wodtke E: Temperature adaptation of biological membranes. Compensation of the molar activity of cytochrome c oxidase in the mitochondrial energy-transducing membrane during thermal acclimation of the carp (Cyprinus carpio L.). Biochim Biophys Acta 1981, 640:710-720.

33. Hulbert AJ, Else PL: Basal metabolic rate: history, composition, regulation, and usefulness. Physiol Biochem Zool 2004, 77:869-876.

34. Clausen T, Van Hardeveld C, Everts ME: Significance of cation transport in control of energy metabolism and thermogenesis. Physiol Rev 1991 71:733-774

35. Wu BJ, Else $\mathrm{PL}$, Storlien $\mathrm{LH}$, Hulbert AJ: Molecular activity of $\mathrm{Na}^{+} / \mathrm{K}^{+}$-ATPase from different sources is related to the packing of membrane lipids. J Exp Biol 2001, 204:4271-4280.

36. Mitchell P: Coupling of phosphorylation to electron and hydrogen transfer by a chemi-osmotic type of mechanism. Nature 1961, 191:144-148.

37. Abrahams JP, Leslie AG, Lutter R, Walker JE: Structure at $2.8 \AA$ resolution of F1-ATPase from bovine heart mitochondria. Nature 1994, 370:621-628.

38. Wojtczak L, Bogucka K, Duszynski J, Zablocka B, Zolkiewska A: Regulation of mitochondrial resting state respiration: slip, leak, heterogeneity? Biochim Biophys Acta 1990, 1018:177-181.

39. Azzu V, Brand MD: The on-off switches of the mitochondrial uncoupling proteins. Trends Biochem Sci 2010, 35:298-307.

40. Brookes PS, Hulbert AJ, Brand MD: The proton permeability of liposomes made from mitochondrial inner membrane phospholipids: no effect of fatty acid composition. Biochim Biophys Acta 1997, 1330:157-164.

41. Brookes PS, Rolfe DF, Brand MD: The proton permeability of liposomes made from mitochondrial inner membrane phospholipids: comparison with isolated mitochondria. J Membr Biol 1997, 155:167-174.

42. Beck V, Jaburek M, Demina T, Rupprecht A, Porter RK, Jezek P, Pohl EE: Polyunsaturated fatty acids activate human uncoupling proteins 1 and 2 in planar lipid bilayers. FASEB J 2007, 21:1137-1144.

43. Murphy MP, Echtay KS, Blaikie FH, Asin-Cayuela J, Cocheme HM, Green K, Buckingham JA, Taylor ER, Hurrell F, Hughes G, Miwa S, Cooper CE, Svistunenko DA, Smith RA, Brand MD: Superoxide activates uncoupling proteins by generating carbon-centered radicals and initiating lipid peroxidation: studies using a mitochondria-targeted spin trap derived from alpha-phenyl-N-tert-butylnitrone. J Biol Chem 2003, 278:48534-48545.

44. Brand MD: Uncoupling to survive? The role of mitochondrial inefficiency in ageing. Exp Gerontol 2000, 35:811-820.

45. Herrero $A$, Barja $\mathrm{G}: \mathrm{H}_{2} \mathrm{O}_{2}$ production of heart mitochondria and aging rate are slower in canaries and parakeets than in mice: sites of free radical generation and mechanisms involved. Mech Ageing Dev 1998, 103:133-146.

46. Bevilacqua L, Ramsey JJ, Hagopian K, Weindruch R, Harper ME: Long-term caloric restriction increases UCP3 content but decreases proton leak and reactive oxygen species production in rat skeletal muscle mitochondria. Am J Physiol Endocrinol Metab 2005, 289:E429-E438.

47. Ramsey JJ, Harper ME, Humble SJ, Koomson EK, Ram JJ, Bevilacqua L, Hagopian K: Influence of mitochondrial membrane fatty acid composition on proton leak and $\mathrm{H}_{2} \mathrm{O}_{2}$ production in liver. Comp Biochem Physiol B Biochem Mol Biol 2005, 140:99-108.

48. Mookerjee SA, Divakaruni AS, Jastroch M, Brand MD: Mitochondrial uncoupling and lifespan. Mech Ageing Dev 2010, 131:463-472.

49. Pamplona R, Prat J, Cadenas S, Rojas C, Perez-Campo R, Lopez Torres M, Barja G: Low fatty acid unsaturation protects against lipid peroxidation in liver mitochondria from long-lived species: the pigeon and human case. Mech Ageing Dev 1996, 86:53-66.

50. Pamplona R, Portero-Otin M, Ruiz C, Gredilla R, Herrero A, Barja G: Double bond content of phospholipids and lipid peroxidation negatively correlate with maximum longevity in the heart of mammals. Mech Ageing Dev 2000, 112:169-183.

51. AnAge database. http://genomics.senescence.info/species.

52. Hulbert AJ, Turner N, Hinde J, Else P, Guderley H: How might you compare mitochondria from different tissues and different species? J Comp Physiol B 2006, 176:93-105.

53. Geiser F: Influence of polyunsaturated and saturated dietary lipids on adipose tissue, brain and mitochondrial membrane fatty acid composition of a mammalian hibernator. Biochim Biophys Acta 1990 1046:159-166.

54. Valencak TG, Ruf T: Phospholipid composition and longevity: lessons from Ames dwarf mice. Age (Dordr) 2013, 35:2303-2313.

55. Geiser F: The effect of unsaturated and saturated dietary lipids on the pattern of daily torpor and the fatty acid composition of tissues and membranes of the deer mouse Peromyscus maniculatus. J Comp Physiol B 1991, 161:590-597.

56. Winczura A, Zdzalik D, Tudek B: Damage of DNA and proteins by major lipid peroxidation products in genome stability. Free Radic Res 2012, 46:442-459

57. Pamplona R, Portero-Otin M, Riba D, Ruiz C, Prat J, Bellmunt MJ, Barja G Mitochondrial membrane peroxidizability index is inversely related to maximum life span in mammals. J Lipid Res 1998, 39:1989-1994.

58. DeLany JP, Windhauser MM, Champagne CM, Bray GA: Differential oxidation of individual dietary fatty acids in humans. Am J Clin Nutr 2000, 72:905-911.

59. Esterbauer H, Schaur RJ, Zollner H: Chemistry and biochemistry of 4hydroxynonenal, malonaldehyde and related aldehydes. Free Radic Biol Med 1991, 11:81-128.

60. Richard D, Kefi K, Barbe U, Bausero P, Visioli F: Polyunsaturated fatty acids as antioxidants. Pharmacol Res 2008, 57:451-455.

61. Andziak B, O'Connor TP, Qi W, DeWaal EM, Pierce A, Chaudhuri AR, Van Remmen $\mathrm{H}$, Buffenstein R: High oxidative damage levels in the longestliving rodent, the naked mole-rat. Aging Cell 2006, 5:463-471.

62. Andziak B, Buffenstein R: Disparate patterns of age-related changes in lipid peroxidation in long-lived naked mole-rats and shorter-lived mice. Aging Cell 2006, 5:525-532.

63. Buffenstein $\mathrm{R}$ : The naked mole-rat: a new long-living model for human aging research. J Gerontol A Biol Sci Med Sci 2005, 60:1369-1377.

64. Lambert AJ, Boysen HM, Buckingham JA, Yang T, Podlutsky A, Austad SN, Kunz $\mathrm{TH}$, Buffenstein R, Brand MD: Low rates of hydrogen peroxide production by isolated heart mitochondria associate with long maximum lifespan in vertebrate homeotherms. Aging Cell 2007, 6:607-618.

65. Mitchell TW, Buffenstein R, Hulbert AJ: Membrane phospholipid composition may contribute to exceptional longevity of the naked mole-rat (Heterocephalus glaber): a comparative study using shotgun lipidomics. Exp Gerontol 2007, 42:1053-1062.

66. Brosche T, Platt D: The biological significance of plasmalogens in defense against oxidative damage. Exp Gerontol 1998, 33:363-369.

67. Zoeller RA, Morand $\mathrm{OH}$, Raetz CR: A possible role for plasmalogens in protecting animal cells against photosensitized killing. J Biol Chem 1988 263:11590-11596.

68. Schmid PC, Deli E, Schmid HH: Generation and remodeling of phospholipid molecular species in rat hepatocytes. Arch Biochem Biophys 1995, 319:168-176.

69. Frank CL: The influence of dietary fatty acids on hibernation by golden-mantled ground squirrels (Spermophilus lateralis). Physiol Zool 1992, 65:906-920. 
70. Geiser F, Baudinette RV: The daily torpor and thermoregulation in the small dasyurid marsupials Planigale gilesi and Ningaui yvonneae. Aust J Zool 1988, 36:473-481

71. Geiser F, Kenagy GJ: Polyunsaturated lipid diet lengthens torpor and reduces body temperature in a hibernator. Am J Physiol 1987, 252:R897-R901.

72. Aloia RC: Lipid, fluidity, and functional studies of the membranes of hibernating mammals. In Advances in Membrane Fluidity. Edited by Aloia RC, Curtain CC, Gordon LM. New York: Alan R Liss; 1988:1-39.

73. Paradies G, Ruggiero FM, Dinoi P, Petrosillo G, Quagliariello E: Decreased cytochrome oxidase activity and changes in phospholipids in heart mitochondria from hypothyroid rats. Arch Biochem Biophys 1993, 307:91-95.

74. Ruf T, Arnold W: Effects of polyunsaturated fatty acids on hibernation and torpor: a review and hypothesis. Am J Physiol Regul Integr Comp Physiol 2008, 294:R1044-R1052.

75. Gerson AR, Brown JC, Thomas R, Bernards MA, Staples JF: Effects of dietary polyunsaturated fatty acids on mitochondrial metabolism in mammalian hibernation. J Exp Biol 2008, 211:2689-2699.

76. Brown JC, Chung DJ, Belgrave KR, Staples JF: Mitochondrial metabolic suppression and reactive oxygen species production in liver and skeletal muscle of hibernating thirteen-lined ground squirrels. Am J Physiol Regul Integr Comp Physiol 2012, 302:R15-R28.

77. Price ER, Valencak TG: Changes in fatty acid composition during starvation in vertebrates: mechanisms and questions. In Comparative Physiology of Fasting, Starvation, and Food Limitation. New York, Dordrecht, London: Springer Heidelberg; 2012:237-255. ISBN 978-3-642-29055-8 doi:10.1007/978-3-642-29056-5.

78. Schlame M, Brody S, Hostetler KY: Mitochondrial cardiolipin in diverse eukaryotes. Comparison of biosynthetic reactions and molecular acyl species. Eur J Biochem 1993, 212:727-735.

79. Pfeiffer K, Gohil V, Stuart RA, Hunte C, Brandt U, Greenberg ML, Schagger H: Cardiolipin stabilizes respiratory chain supercomplexes. J Biol Chem 2003, 278:52873-52880.

80. Pebay-Peyroula E, Dahout-Gonzalez C, Kahn R, Trezeguet V, Lauquin GJ, Brandolin G: Structure of mitochondrial ADP/ATP carrier in complex with carboxyatractyloside. Nature 2003, 426:39-44.

81. Claypool SM, Oktay Y, Boontheung P, Loo JA, Koehler CM: Cardiolipin defines the interactome of the major ADP/ATP carrier protein of the mitochondrial inner membrane. J Cell Biol 2008, 182:937-950.

82. Osman C, Voelker DR, Langer T: Making heads or tails of phospholipids in mitochondria. J Cell Biol 2011, 192:7-16.

83. Gonzalvez F, Schug ZT, Houtkooper RH, Mackenzie ED, Brooks DG, Wanders RJ, Petit PX, Vaz FM, Gottlieb E: Cardiolipin provides an essential activating platform for caspase-8 on mitochondria. J Cell Biol 2008, 183:681-696.

84. Choi SY, Gonzalvez F, Jenkins GM, Slomianny C, Chretien D, Arnoult D, Petit PX, Frohman MA: Cardiolipin deficiency releases cytochrome $c$ from the inner mitochondrial membrane and accelerates stimuli-elicited apoptosis. Cell Death Differ 2007, 14:597-606.

85. Paradies G, Paradies V, Ruggiero FM, Petrosillo G: Changes in the mitochondrial permeability transition pore in aging and age-associated diseases. Mech Ageing Dev 2013, 134:1-9.

86. Petrosillo G, De Benedictis V, Ruggiero FM, Paradies G: Decline in cytochrome c oxidase activity in rat-brain mitochondria with aging. Role of peroxidized cardiolipin and beneficial effect of melatonin. J Bioenerg Biomembr 2013, 45:431-440.

87. Zhou J, Zhong Q, Li G, Greenberg ML: Loss of cardiolipin leads to longevity defects that are alleviated by alterations in stress response signaling. J Biol Chem 2009, 284:18106-18114.

88. Gruber J, Ng LF, Fong S, Wong YT, Koh SA, Chen CB, Shui G, Cheong WF, Schaffer S, Wenk MR, Halliwell B: Mitochondrial changes in ageing Caenorhabditis elegans - what do we learn from superoxide dismutase knockouts? PLoS One 2011, 6:e19444.

89. Lee HJ, Mayette J, Rapoport SI, Bazinet RP: Selective remodeling of cardiolipin fatty acids in the aged rat heart. Lipids Health Dis 2006, 5:2.

90. Jobson RW, Nabholz B, Galtier N: An evolutionary genome scan for longevity-related natural selection in mammals. Mol Biol Evol 2010, 27:840-847.

91. Speakman JR: Correlations between physiology and lifespan - two widely ignored problems with comparative studies. Aging Cell 2005, 4:167-175.

92. R project. www.R-project.org.
93. Felsenstein J: Phylogenies and the comparative method. Am Nat 1985, 125:1-15.

94. Sohal RS, Weindruch R: Oxidative stress, caloric restriction, and aging. Science 1996, 273:59-63.

95. Lambert AJ, Portero-Otin M, Pamplona R, Merry BJ: Effect of ageing and caloric restriction on specific markers of protein oxidative damage and membrane peroxidizability in rat liver mitochondria. Mech Ageing Dev 2004, 125:529-538

96. Faulks SC, Turner N, Else PL, Hulbert AJ: Calorie restriction in mice: effects on body composition, daily activity, metabolic rate, mitochondrial reactive oxygen species production, and membrane fatty acid composition. J Gerontol A Biol Sci Med Sci 2006, 61:781-794.

97. Shmookler Reis RJ, Xu L, Lee H, Chae M, Thaden JJ, Bharill P, Tazearslan C, Siegel E, Alla R, Zimniak P, Ayyadevara S: Modulation of lipid biosynthesis contributes to stress resistance and longevity of $C$. elegans mutants. Aging (Albany NY) 2011, 3:125-147.

98. Abbott SK, Else PL, Atkins TA, Hulbert AJ: Fatty acid composition of membrane bilayers: importance of diet polyunsaturated fat balance. Biochim Biophys Acta 1818, 2012:1309-1317.

99. Hu FB, Willett WC: Optimal diets for prevention of coronary heart disease. JAMA 2002, 288:2569-2578.

100. Hu FB, Stampfer MJ, Manson JE, Ascherio A, Colditz GA, Speizer FE, Hennekens $\mathrm{CH}$, Willett WC: Dietary saturated fats and their food sources in relation to the risk of coronary heart disease in women. Am J Clin Nutr 1999, 70:1001-1008.

101. Wilson PW: Established risk factors and coronary artery disease: the Framingham study. Am J Hypertens 1994, 7:7S-12S.

doi:10.1186/2046-2395-3-3

Cite this article as: Valencak and Azzu: Making heads or tails of mitochondrial membranes in longevity and aging: a role for comparative studies. Longevity \& Healthspan 2014 3:3.

\section{Submit your next manuscript to BioMed Central and take full advantage of:}

- Convenient online submission

- Thorough peer review

- No space constraints or color figure charges

- Immediate publication on acceptance

- Inclusion in PubMed, CAS, Scopus and Google Scholar

- Research which is freely available for redistribution 